\title{
CARCINOSARCOMA UTERINO CON EXTENSIÓN PÉLVICA: PRESENTACIÓN DE UN CASO Y REVISIÓN DE LA LITERATURA
}

\section{Uterine carcinosarcoma with extension to the pelvis: Case presentation and review of the literature}

César Redondo-Bermúdez, $M D^{\prime}$; Liney Mendoza-Suárez, $M D^{2}$;

Karina Ruiz-Cáez, $M^{2}$; Jorge Gómez-Villa

Recibido: enero 28/14 - Aceptado: diciembre 15/14

\section{RESUMEN}

Objetivo: se presenta un caso de carcinosarcoma uterino para hacer una revisión de la literatura publicada acerca de la epidemiologia, el diagnóstico, tratamiento y pronóstico de esta neoplasia.

Materiales y métodos: se presenta el caso de una paciente de 73 años de edad, quien consultó a un centro de referencia para atención ginecoobstétrica en Cartagena (Colombia), por sangrado vaginal, pérdida de peso y masa pélvica. El análisis microscópico reveló un carcinosarcoma endometrial/ tumor mülleriano mixto maligno. Se realizó una búsqueda sistemática en las bases de datos Medline vía PubMed, Hinari, Imbiomed, ScienceDirect y EBSCO, utilizando los términos "Carcinosarcoma", "Neoplasias uterinas", "Útero". Se buscaron estudios tipo reporte de casos, revisiones de temas u otros estudios publicados en los últimos diez años, en inglés o español.

Resultados: se encontraron 350 títulos de los cuales un total de 20 artículos estaban relaciona-

1 Médico Patólogo. Docente, Sección de Patología, Departamento de Diagnóstico, Facultad de Medicina, Universidad de Cartagena (Colombia).

2 Médica. Residente de Patología, Facultad de Medicina, Universidad de Cartagena (Colombia).

3 Estudiante de Pregrado, Facultad de Medicina, Universidad de Cartagena (Colombia).jorkgovi@hotmail.com dos directamente con el tema, de estos se seleccionaron 18 que correspondieron a ocho reportes de casos, siete revisiones de temas, dos cohortes retrospectivas y un ensayo clínico. El carcinosarcoma uterino es una neoplasia poco frecuente que afecta especialmente a mujeres posmenopáusicas. Histopatológicamente se observa una combinación de cambios carcinomatosos y sarcomatosos; el tratamiento estándar es quirúrgico. La profundidad de la invasión miometrial ha sido sugerida como factor pronóstico muy importante, y en un $50 \%$ de los casos presenta extensión extrauterina que se asocia con una supervivencia general muy pobre. Conclusiones: el carcinosarcoma es una neoplasia de baja frecuencia caracterizada por su población celular bifásica y mal pronóstico.

Palabras clave: carcinosarcoma, neoplasias uterinas, útero.

\section{ABSTRACT}

Objective: Presentation of a case of uterine carcinosarcoma and review of the literature on the epidemiology, diagnosis, treatment and prognosis of this neoplasm.

Materials and methods: A 73 year-old female patient presenting with vaginal bleeding, weight loss and a pelvic mass to a referral centre for gynaecological and obstetrical care in Cartagena, 
Colombia. The microscopic examination revealed a mixed malignant endometrial carcinosarcoma/ müllerian tumor. A systematic search was conducted in the Medline via PubMed, Hinari, Imbiomed, ScienceDirect and Ebsco databases, using the terms "carcinosarcoma", "uterine neoplasms", "uterus". The search included case reports, topic reviews or other studies published within the past 10 years, both in English and Spanish.

Results: Overall, 350 titles were found, 20 of which were directly related to the topic. Of these, 18 were selected, including 8 case reports, 7 topic reviews, 2 retrospective cohorts, and 1 clinical trial. Uterine carcinosarcoma is an infrequent neoplasm affecting post-menopausal women in particular. The histopathology shows a combination of carcinomatous and sarcomatous changes. The standard treatment is surgery. It has been suggested that the depth of invasion into de myometrium is a very important prognostic factor, and extra-uterine extension is found in $50 \%$ of cases. It is generally associated with very poor survival.

Conclusions: Carcinosarcoma is an infrequent neoplasm characterized by biphasic cell population and poor prognosis.

Key words: Carcinosarcoma, uterine neoplasms, uterus.

\section{INTRODUCCIÓN}

Los tumores müllerianos mixtos malignos (TMMM) son adenocarcinomas endometriales en los que se ha producido una diferenciación mesenquimatosa maligna. Se les llama tumores mixtos porque presentan un componente epitelial (carcinomatoso) y estromal (sarcomatoso), el segundo componente tiende a diferenciarse en diversos elementos mesodérmicos malignos, que pueden ser de origen muscular, cartilaginoso, osteoide, etc. $(1,2)$. Son neoplasias uterinas infrecuentes que derivan de los conductos paramesonéfricos o de Müller. Durante el periodo embrionario estos conductos dan origen al endometrio (epitelio mülleriano), miometrio y serosa uterina (mesénquima mülleriano) (3). Dentro de este grupo de TMMM, se encuentra el carcinosarcoma. El término carcinosarcoma se utilizó por primera vez por Virchow en 1864 para describir los tumores con elementos carcinomatosos y sarcomatosos (4), representa menos del $5 \%$ de todas las neoplasias uterinas (5).

Existe controversia acerca de la histogénesis de los TMMM de tracto genital femenino; han sido propuestas cuatro teorías denominadas "colisión”, "combinación”, “composición” y "carcinoma metaplásico”. La primera teoría postula que hay un origen celular bifásico con dos proliferaciones neoplásicas sincrónicas, separadas, que luego se unen formando un solo tumor. La teoría de la combinación sugiere que los componentes epiteliales y sarcomatosos comparten un mismo origen. La teoría de la composición plantea un origen monoclonal, donde el componente mesenquimático es producto de un fenómeno de metaplasia del tejido carcinomatoso (3). Por último, la teoría del carcinoma metaplásico postula un origen común con metaplasia de ambos componentes resultando en una diferencia neoplásica divergente. Esta última teoría ha ganado mayor aceptación por los resultados de algunos estudios inmunofenotípicos (6).

La estadificación del carcinosarcoma uterino fue realizada por la International Federation of Gynecologists and Obstetricians (FIGO) con los mismos criterios quirúrgicos de estadificación utilizados para carcinomas del cuerpo uterino (7). Dada la baja frecuencia de este tipo de neoplasia, los rasgos histopatológicos y los retos diagnósticos que representa para el ginecólogo, oncólogo, médico internista, médico patólogo o médico general, se presenta un caso reportado por el servicio de Patología de la Clínica Maternidad Rafael Calvo de la ciudad de Cartagena de Indias (Colombia), con el objetivo de realizar una revisión de la literatura publicada acerca de las características epidemiológicas, clínicas, histopatológicas, de tratamiento y pronósticas. 


\section{CASO CLIINICO}

Paciente de 73 años, que consultó a la Clínica Maternidad Rafael Calvo de Cartagena (Colombia), institución de segundo nivel de complejidad, la cual es centro de referencia para la atención ginecoobstétrica en el departamento de Bolívar, que atiende a pacientes del aseguramiento subsidiado por el Estado en el régimen de seguridad social en Colombia. Fue vista con cuadro clínico de seis meses de evolución caracterizado por sangrado vaginal, astenia, adinamia y pérdida de peso no cuantificada. Antecedentes personales: hipertensión arterial en tratamiento. Al examen físico presentaba palidez mucocutánea generalizada y masa en hipogastrio. La ecografía abdominal total reportó útero aumentado de tamaño con masa $10 \times 8 \mathrm{~cm}$, y otra en el fondo del saco de Douglas, de límites mal definidos, de 13 x $10 \mathrm{~cm}$. Se diagnosticó tumor maligno de ovario, motivando valoración por ginecología oncológica. Se lleva a cirugía, donde se encuentra masa que ocupa fondo de saco de Douglas que compromete el recto y la pared pélvica, con múltiples vesículas y tabiques; útero aumentado de tamaño, reblandecido, con infiltración de tumor en cérvix; los ovarios, de aspecto atrófico, están libres de tumor (figuras 1 y 2). Se realiza histerectomía abdominal total, salpingo-oforectomía bilateral, omentectomía parcial, apendicetomía y liberación de adherencias en pelvis y útero. Durante el acto quirúrgico requirió soporte inotrópico, transfusión sanguínea, colocación de catéter central y monitorización posquirúrgica en cuidados intermedios con diagnóstico de shock hipovolémico más sarcoma uterino con extensión a pelvis. Se realiza estudio histológico que reporta útero con carcinosarcoma endometrial/tumor mülleriano mixto maligno, en estadio IV-B según la estadificación FIGO, infiltrante (tamaño tumoral: 13 x $12 \times 5 \mathrm{~cm}$ ), con invasión linfovascular, sin evidencia de componente mesenquimal heterólogo (figura 3). Resección de lesión pélvica, evidencia de metástasis por carcinosarcoma, fragmentado (masa mayor tamaño 15 x 11 x $4 \mathrm{~cm}$ y masa menor tamaño $4 \times 3 \times 2 \mathrm{~cm}$ ). La paciente inicia seguimiento por ginecología oncológica, y en conjunto con familiares deciden no realizar tratamiento adyuvante, se mantiene estable y sin recaída hasta los tres meses de seguimiento; seis meses después fallece.

\section{MATERIALES Y MÉTODOS}

Se realizó una búsqueda sistemática de la literatura en Medline vía PubMed, Hinari, Imbiomed, Springerlink, ScienceDirect y EBSCO usando los términos: "Carcinosarcoma", "Neoplasias uterinas", "Útero". Se buscaron estudios publicados en los
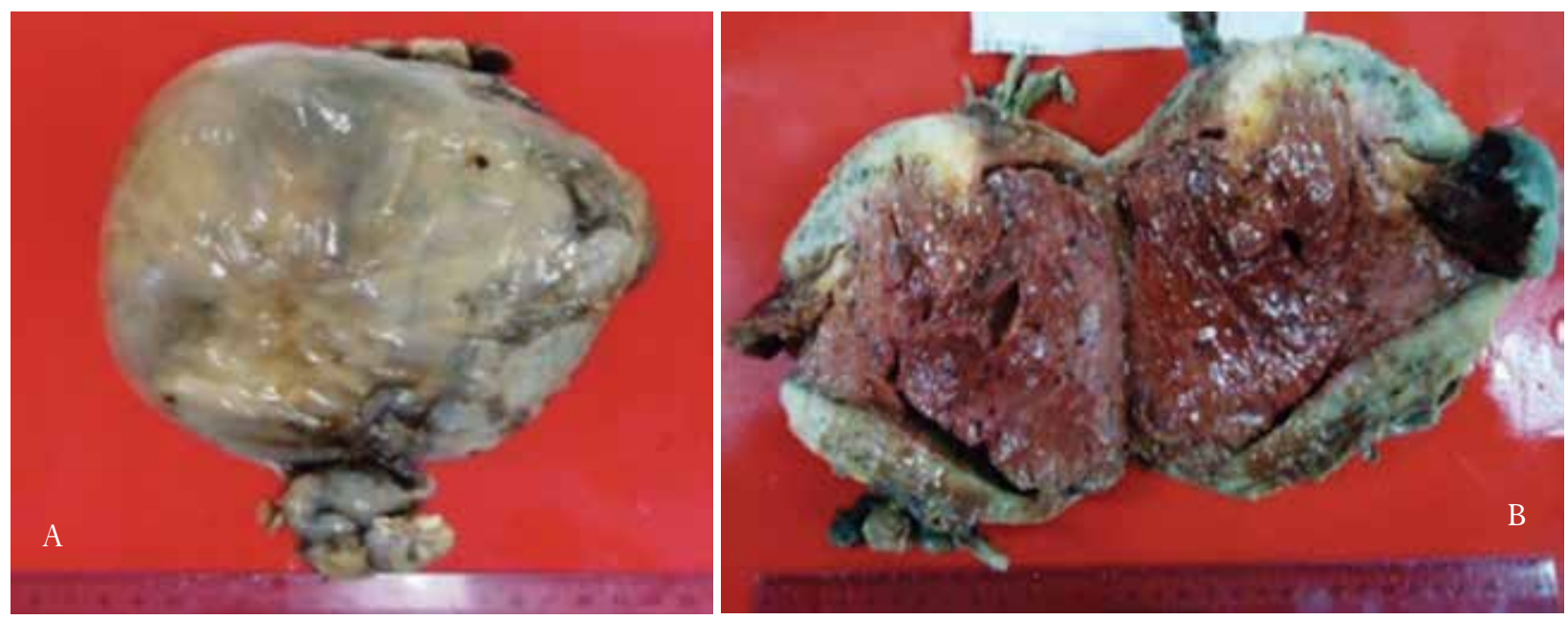

Figura 1. A. Útero aumentado de tamaño, reblandecido, de $15 \times 13$ x $5 \mathrm{~cm}$. B. Corte de útero con anexos. Se observa masa de 12 x $9 \mathrm{~cm}$ abortada por orificio cervical externo e infiltración de la pared miometrial. 

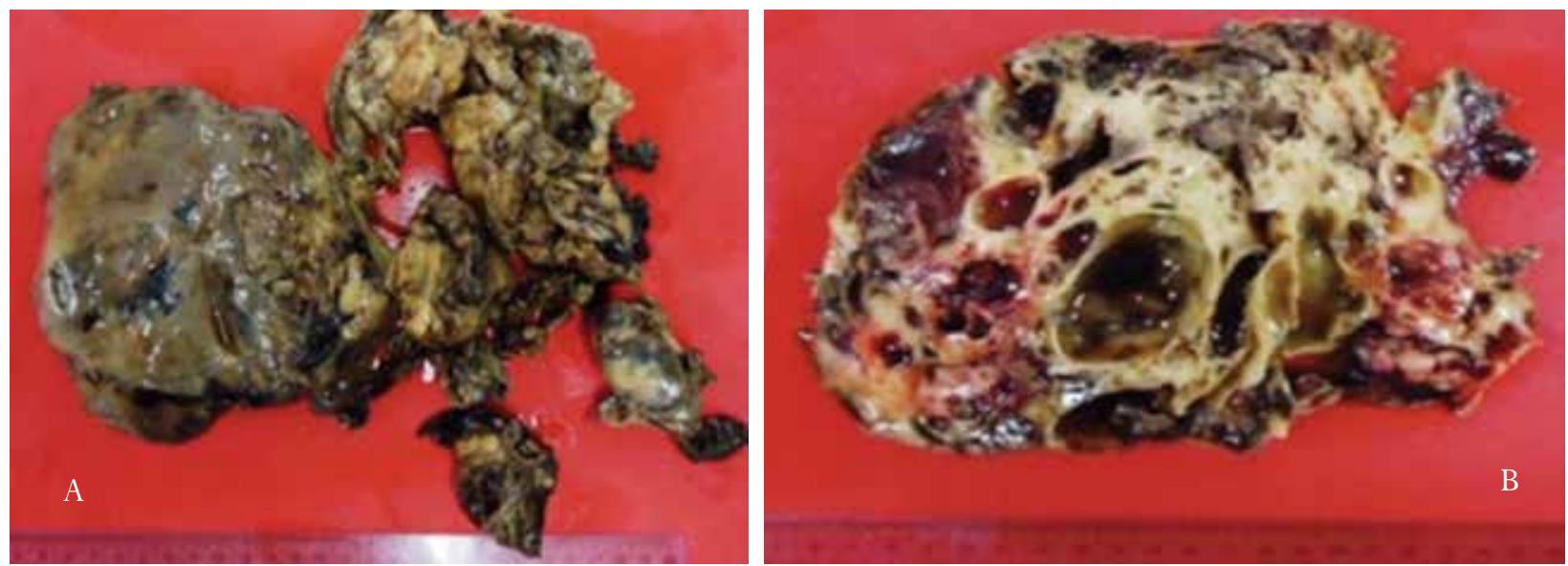

Figura 2. A. Masa pélvica fragmentada. La mayor mide $15 \times 11$ x $4 \mathrm{~cm}$ y la menor 4 x $3 \times 2 \mathrm{~cm}$. B. Cortes de masa pélvica de mayor tamaño. Superficie sólida y quística con necrosis y áreas de hemorragia.

últimos diez años, en inglés o español, que trataran sobre la epidemiología, el diagnóstico, tratamiento y pronóstico de este tipo de neoplasia.

Aspectos éticos. Se solicitó autorización a la paciente para publicar las fotos y se garantizó la confidencialidad de la información para proteger sus derechos.

\section{RESULTADOS}

Se encontraron 350 artículos, de los cuales un total de 20 estaban relacionados directamente con el tema, de estos se seleccionaron 18 que correspondie- ron a ocho reportes de casos, siete revisiones de temas, dos cohortes retrospectivas y un ensayo clínico.

Epidemiología. Su incidencia es de 2 por 100.000 mujeres por año (5). Se estiman entre 1.000-1.500 casos nuevos cada año. Se presentan principalmente en pacientes posmenopáusicas, con una media de edad entre 60 a 70 años (8).

El carcinosarcoma representa el $50 \%$ de los sarcomas uterinos, seguidos de leiomiosarcomas (30\%), sarcomas del estroma endometrial (10\%) y adenosarcomas (8\%), siendo el resto de variedades histológicas una verdadera rareza $(9,10)$.
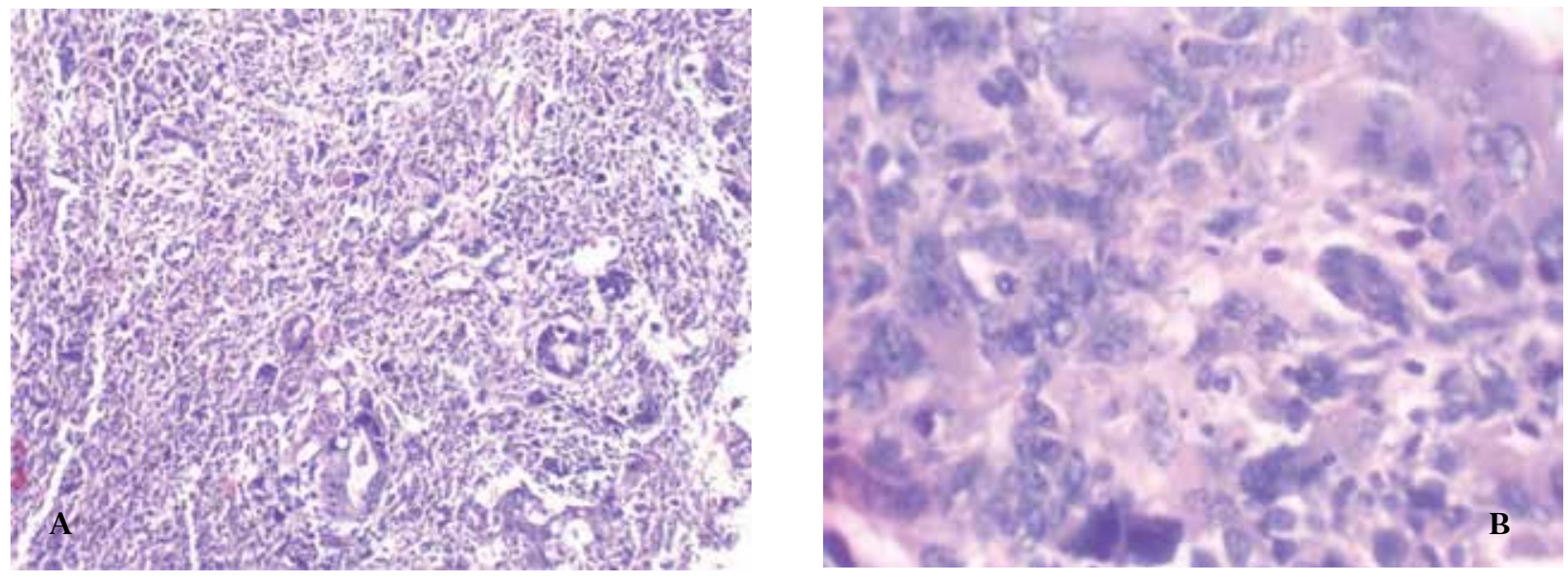

Figura 3. A. Panorámica de lesión tumoral maligna bifásica constituida por un componente de linaje epitelial y mesenquimal sin componente heterólogo. B. Componente mesenquimal constituido por células grandes, algunas redondeadas, núcleos pleomórficos y multilobulados, con nucleolos conspicuos y citoplasma eosinofílico. 
Características clínicas. La presentación clínica de los carcinosarcomas uterinos es similar a la de los adenocarcinomas de endometrio, generalmente se manifiesta con metrorragia posmenopáusica o masas uterinas que en muchos casos protruyen por el orificio cervical externo (2). Los signos pueden incluir ascitis, plastrón omental y carcinomatosis peritoneal (11). Se ha propuesto que el sangrado uterino es el síntoma más común del carcinosarcoma espontáneo, mientras que el síntoma más común del asociado a irradiación es la distensión abdominal y el dolor (8). La ubicación más frecuente es en el cuerpo del útero, particularmente en la pared posterior en la región del fundus; sin embargo, pueden surgir en ubicaciones extrauterinas, los sitios más comunes son el ovario y estructuras de la pelvis. Es común la extensión dentro de la pelvis, con invasión vascular y linfática, produciendo metástasis a distancia (1). Por ejemplo, se informó de un caso de metástasis a calcáneo (12). La tomografía computarizada del tórax, el abdomen y la pelvis pueden ser útiles en la estatificación del tumor (11).

Características histopatológicas. Macroscópicamente se presenta como masa polipoide, de gran tamaño, consistencia blanda, que involucra el endometrio y el miometrio; con frecuencia hay focos de necrosis y hemorragias (1). Microscópicamente, la característica fundamental es la combinación de los cambios carcinomatosos y sarcomatosos, resultando en cambios bifásicos muy característicos. El componente carcinomatoso es generalmente de tipo glandular, y el endometrial son células claras o sero-papilares. Es un tumor poco diferenciado. Se puede observar, además, la presencia de células escamosas indiferenciadas y pequeños focos de tejido neuroectodérmico. La aparición de tejido sarcomatoso es la base para la división en dos variedades: la homóloga y la heteróloga. Al comienzo el estroma lo constituyen células redondeadas que recuerdan el estroma endometrial, o células alargadas que recuerdan el leiomiosarcoma o fibrosarcoma. El análisis inmunohistoquímico de diversos antígenos humanos se ha realizado para evaluar la naturaleza y la histogénesis de diferentes componentes malignos del carcinosarcoma uterino (13). La identificación de estriaciones o de marcadores de músculo esquelético por inmunohistoquímica es necesaria para poder documentar la presencia de un componente rabdomiosarcomatoso (1). La clasificación de este tipo de tumor depende de la diferenciación sarcomatosa, es decir, si los elementos integrantes de tipo sarcomatoso imitan a los tejidos extrauterinos (células musculares estriadas, cartílago, tejido adiposo y hueso), se denomina TMMM heterólogo. Por el contrario, si los componentes del mesénquima consisten en una diferenciación maligna endometrial o de músculo liso, se utiliza el término de carcinosarcoma o TMMM homólogo. En fases tardías del tumor los elementos heterólogos están presentes $(1,2)$.

Marcadores pronósticos. La evaluación de la actividad proliferativa del tumor ha sido informada como un factor pronóstico relevante en diversos tumores malignos ginecológicos, incluyendo cáncer de endometrio. Se ha informado una diferencia significativa en el índice de clasificación a partir del marcador histoquímico MIB-1 entre las áreas carcinomatosas $(51,8 \%)$ y sarcomatosas $(28,6 \%)$ en el carcinosarcoma del tracto genital femenino, que fue un índice útil para evaluar el pronóstico de las mujeres afectadas por TMMM uterinas (14). El Ki-67 se expresa en el ciclo celular en la proliferación de células solamente. Varios estudios revelaron que la expresión elevada de Ki-67 tiene un valor pronóstico negativo en varios tipos de tumores malignos humanos. El p53 es una proteína supresora de tumores que pueden inducir la apoptosis; en general, la sobreexpresión de estos marcadores determina el comportamiento agresivo en muchos tipos de tumores malignos, sin embargo, es importante resaltar que estos parámetros pueden no ser indicadores de supervivencia (15).

Tratamiento. La histerectomía abdominal total con salpingectomía y linfadenectomía pélvica es el tratamiento estándar (5). No obstante, el tratamiento quirúrgico es un tema en ocasiones 
controvertido, que implica dos aspectos: el estudio ganglionar y la omentectomía. El estudio ganglionar es especialmente importante en los carcinosarcomas, debido a que en el momento del diagnóstico presentan una incidencia de metástasis ganglionares pélvicas de hasta el $45 \%$. En el cáncer de endometrio se ha consideró, desde hace muchos años, que el resultado en cuanto a supervivencia no se modificaba si la paciente efectuaba una histerectomía total con anexectomía bilateral seguida de radioterapia (10). Sin embargo, la citorreducción óptima de los pacientes con carcinoma avanzado uterino ha demostrado una ventaja de supervivencia especialmente en estadios IV-B (16).

Hay poca evidencia para apoyar el beneficio del tratamiento adyuvante en la enfermedad avanzada, por lo general se recomienda el uso de radioterapia o quimioterapia adyuvante (8). La radioterapia pélvica adyuvante en estadios I-II se asocia con un beneficio de supervivencia (17). En otros estadios, la radioterapia disminuye la tasa de recidivas pélvicas y metástasis a distancia, pero no mejora la supervivencia $(11,17)$. La radioterapia pélvica no está indicada en pacientes con antecedentes de exposición a radioterapia pélvica previa o si recibieron solo quimioterapia como tratamiento adyuvante (8). Se ha demostrado que la quimioterapia adyuvante con cisplatino e ifosfamida permite una supervivencia del $52 \%$ a cinco años en pacientes con enfermedad en estadios I y II; sin embargo, se presenta recidiva en vagina o cavidad pélvica en un porcentaje del $17 \%$ (11). En los pacientes con enfermedad en estadios III y IV, y $<1 \mathrm{~cm}$ residual, la terapia con cisplatino adyuvante y ifosfamida fue superior a la radioterapia abdominal completa (11).

Pronóstico. Los TMMM son neoplasias altamente agresivas, incluso más que la variante desfavorable del carcinoma endometrial. Actualmente, el factor pronóstico más confiable es la estatificación quirúrgica que ha permitido predecir las consecuencias finales de este tipo de neoplasia.
La profundidad de la invasión miometrial ha sido sugerida como el factor pronóstico más importante. Si el tumor se ha extendido a la serosa del útero o más allá al tiempo de la cirugía, el pronóstico es desalentador (1). A diferencia de los adenocarcinomas de endometrio, en donde el $70 \%$ de los tumores se limita al útero al momento del diagnóstico, el carcinosarcoma en un $50 \%$ de los casos presenta extensión extrauterina que se asocia con una supervivencia general muy pobre (16). La única posibilidad de cura es cuando el tumor está restringido al $50 \%$ del grosor endometrial (1).

Aproximadamente la tercera parte recidivan localmente y otra tercera parte presentarán metástasis a distancia en el curso del primer año (8). Estudios apoyan que el comportamiento de esta neoplasia está íntimamente relacionado con el componente epitelial debido a que invade espacios linfáticos, vasculares y realiza metástasis, mientras que el componente de células fusiformes tiene potencial metastásico muy limitado, en particular en las primeras etapas del tumor (18). Estudios han informado que la sobrevida global estimada a tres años para todos los estadios es de 48,9\% y la sobrevida a 5 años es del 18 al $39 \%(5,18)$.

\section{CONCLUSIÓN}

El carcinosarcoma uterino es una neoplasia poco frecuente, se presenta principalmente en mujeres posmenopáusicas. Está constituido por su población celular bifásica, compuesta por un componente carcinomatoso y sarcomatoso (homólogo). Es una neoplasia agresiva, con una gran capacidad de metástasis a distancia y recidivas locales. El tratamiento estándar es quirúrgico y hay poca claridad acerca de su tratamiento adyuvante. Su sobrevida global a 3 años es menor del 50\%, lo que ratifica la gran agresividad de la neoplasia en estas pacientes.

\section{AGRADECIMIENTOS}

Agradecemos a los docentes y residentes de la sección de patología, Departamento de Diagnóstico, Universidad de Cartagena, por la revisión y los co- 
mentarios sobre el manuscrito; a los profesionales de la salud y personal administrativo del Laboratorio de Citopatología del Caribe, quienes participaron en el abordaje diagnóstico y terapéutico del paciente, y colaboraron de alguna manera con la estructuración de este reporte.

\section{REFERENCIAS}

1. Ávila Aróstegui DL, Amores Carraté J, Bastián Manso L, Arredondo Bruce A. Tumor maligno mülleriano mixto: a propósito de un caso raro. Rev Arch Méd Camagüey. 2010;14.

2. José Gutiérrez Y, Puig Ferrer F, Baquedano Mainar L, Lanzón Laga A, Vicente Arregui S, Ruiz Conde MÁ. Tumores müllerianos mixtos malignos uterinos. Rev Chil Obstet Ginecol. 2011;76:420-6.

3. Siebald CE, Puga SO, Madrid SP. Tumores müllerianos mixtos de cuello uterino. Rev Chil Obstet Ginecol. 2008;73:128-32.

4. Ferrandina G, Zannoni GF, Martinelli E, Vellone V, Prisco MG, Scambia G. Endometrial carcinoma recurring as carcinosarcoma: report of two cases. Pathol Res Pract. 2007;203:677-81.

5. Malca TM, López BA, Santos OC, Álvarez LM. Factores pronósticos para sobrevida en pacientes con carcinosarcoma uterino. Rev Peru Ginecol Obstet. 2013;59:119-24.

6. McCluggage WG. Uterine carcinosarcomas (malignant mixed Mullerian tumors) are metaplastic carcinomas. Int J Gynecol Cancer Off J Int Gynecol Cancer Soc. 2002;12:687-90.

7. Fowler JM, Blessing JA, Burger RA, Malfetano JH. Phase II evaluation of oral trimetrexate in mixed mesodermal tumors of the uterus: a gynecologic oncology group study. Gynecol Oncol. 2002;85:311-4.

8. Kahraman K, Ortac F, Kankaya D, Aynaoglu G. Uterine carcinosarcoma associated with pelvic radiotherapy for sacral chordoma: a case report. Taiwan J Obstet Gynecol. 2012;51:89-92.
9. Solis CJ. Sarcomas uterinos: manejo actual. Rev Chil Obstet Ginecol. 2005;70:332-9.

10. Rovirosa A. Tumores uterinos con componente sarcomatoso: pasado, presente y futuro. Oncol Barc. 2006;29:11-5.

11. Sutton G. Uterine sarcomas 2013. Gynecol Oncol. 2013;130:3-5.

12. Rice BM, Todd NW, Jensen R, Rush SM, Rogers W. Metastatic Calcaneal Lesion Associated with Uterine Carcinosarcoma. J Foot Ankle Surg. 2014;53:364-8. doi: $10.1053 /$ j.

13. Semczuk A, Zakrzewski PK, Forma E, Cygankiewicz AI, Semczuk-Sikora A, Brys M, et al. TGF $\beta$-pathway is down-regulated in a uterine carcinosarcoma: A case study. Pathol Res Pract. 2013;209:740-4. doi: $10.1016 / \mathrm{j}$

14. Semczuk A, Skomra D, Chyzynska M, Szewczuk W, Olcha P, Korobowicz E. Immunohistochemical analysis of carcinomatous and sarcomatous components in the uterine carcinosarcoma: A case report. Pathol Res Pract. 2008;204:203-7.

15. Lee S-J, Kim HS, Kim HS, Chun YK, Hong SR, Lee J-H. Immunohistochemical study of DNA topoisomerase I, p53, and Ki-67 in uterine carcinosarcomas. Hum Pathol. 2007;38:1226-31.

16. Tanner EJ, Leitao MM Jr, Garg K, Chi DS, Sonoda Y, Gardner GJ, et al. The role of cytoreductive surgery for newly diagnosed advanced-stage uterine carcinosarcoma. Gynecol Oncol. 2011;123:548-52.

17. Clayton Smith D, Kenneth Macdonald O, Gaffney DK. The impact of adjuvant radiation therapy on survival in women with uterine carcinosarcoma. Radiother Oncol. 2008;88:227-32.

18. Cimbaluk D, Rotmensch J, Scudiere J, Gown A, Bitterman P. Uterine carcinosarcoma: immunohistochemical studies on tissue microarrays with focus on potential therapeutic targets. Gynecol Oncol. 2007;105:138-44. 